\title{
CHARACTERIZING STRONG ESTIMATES
}

\author{
CHAOYUAN LIU AND JOSEPH ROSENBLATT
}

(Communicated by Jane M. Hawkins)

\begin{abstract}
We describe necessary and sufficient conditions for square functions to map $L^{\infty}$ to $L^{\infty}$ for ergodic averages and Lebesgue derivatives.
\end{abstract}

\section{INTRODUCTION}

Square functions are extensively studied in ergodic theory. It has been proved in [5] and [6] that square functions for ergodic averages and Lebesgue derivatives are of weak type $(1,1)$ and of strong type $(p, p)$ for $1<p<\infty$. It is also proved in [6], [7], and [9] that square functions map $L^{\infty}$ to $B M O$ for Lebesgue derivatives. In particular it has been proved in [12] that the square functions for Lebesgue derivatives map $B M O$ to $B M O$. This is why we concentrate on characterizing the necessary and sufficient conditions for square functions of ergodic averages or Lebesgue derivatives to map $L^{\infty}$ to $L^{\infty}$. We show that this condition for the square function of the successive differences of a sequence $\left(A_{n_{k}}\right)$ of ergodic averages is that $\sum_{k=1}^{\infty}\left(\frac{n_{k+1}-n_{k}}{n_{k+1}}\right)^{2}$ is finite. A similar necessary and sufficient condition can be given for Lebesgue derivatives.

First, we define the square functions for ergodic theory. Let $(X, \mathcal{F}, \mu, T)$ be a dynamical system, and let $T$ be an ergodic transformation from $X$ to $X$. Given $f \in L^{p}=L^{p}(X, \mathcal{F}, \mu)$, let $A_{n} f=\frac{1}{n} \sum_{l=1}^{n} f \circ T^{l}$ be the usual average in ergodic theory.

Definition 1.1. Given a sequence $\left(n_{k}\right)$ of whole numbers, the square function $S f$ is defined as

$$
S f=\left(\sum_{k=1}^{\infty}\left|A_{n_{k+1}} f-A_{n_{k}} f\right|^{2}\right)^{\frac{1}{2}} .
$$

Now, we define the square functions for Lebesgue derivatives.

Definition 1.2. Given a sequence of real numbers $\left(\epsilon_{k}\right)$, for any $x \in \mathbb{R}$, let

$$
D_{k} f(x)=\frac{1}{\epsilon_{k}} \int_{0}^{\epsilon_{k}} f(x+t) d t .
$$

Received by the editors October 5, 2006.

2000 Mathematics Subject Classification. Primary 37A05, 37A50, 26A45; Secondary 28D05.

The second author recognizes the support of the NSF during the preparation of this article. 
Also, for any $x \in \mathbb{R}$, let

$$
S f(x)=\left(\sum_{k=1}^{\infty}\left|D_{k} f(x)-D_{k+1} f(x)\right|^{2}\right)^{\frac{1}{2}} .
$$

We will need to use the partial sums $S_{M}$ of both of these square functions in this article.

\section{Characterizing the Strong estimates For ERgodic AVERAges}

In this section, we give necessary and sufficient conditions for the square function of differences of ergodic averages to map $L^{\infty}$ to $L^{\infty}$.

Theorem 2.1. For a dynamical system $(X, \Sigma, \mu, T)$, if $\sum_{k=1}^{\infty}\left|\frac{n_{k+1}-n_{k}}{n_{k+1}}\right|^{2}<\infty$, then $S$ maps $L^{\infty}$ to $L^{\infty}$. Also, if

$$
C=2\left\{\sum_{k=1}^{\infty}\left|\frac{n_{k+1}-n_{k}}{n_{k+1}}\right|^{2}\right\}^{\frac{1}{2}},
$$

then for all $f \in L^{\infty},\|S f\|_{\infty} \leq C\|f\|_{\infty}$.

Proof. Let $T$ be a measure preserving transformation from $X$ to $X$ and let $f \in L^{\infty}$. We have

$$
\begin{aligned}
S f(x) & =\left(\sum_{k=1}^{\infty}\left(A_{n_{k+1}} f(x)-A_{n_{k}} f(x)\right)^{2}\right)^{\frac{1}{2}} \\
& =\left(\sum_{k=1}^{\infty}\left(\frac{1}{n_{k+1}} \sum_{j=1}^{n_{k+1}} f\left(T^{j}(x)\right)-\frac{1}{n_{k}} \sum_{j=1}^{n_{k}} f\left(T^{j}(x)\right)\right)^{2}\right)^{\frac{1}{2}} \\
& =\left(\sum_{k=1}^{\infty}\left(\frac{1}{n_{k+1}} \sum_{j=n_{k}+1}^{n_{k+1}} f\left(T^{j}(x)\right)-\left(\frac{1}{n_{k}}-\frac{1}{n_{k+1}}\right) \sum_{j=1}^{n_{k}} f\left(T^{j}(x)\right)\right)^{2}\right)^{\frac{1}{2}} \\
& \leq\left(\sum_{k=1}^{\infty}\left(\frac{1}{n_{k+1}} \sum_{j=n_{k}+1}^{n_{k+1}} f\left(T^{j}(x)\right)\right)^{2}\right)^{\frac{1}{2}}+\left(\sum_{k=1}^{\infty}\left(\left(\frac{1}{n_{k}}-\frac{1}{n_{k+1}}\right) \sum_{j=1}^{n_{k}} f\left(T^{j}(x)\right)\right)^{2}\right)^{\frac{1}{2}} \\
& \leq\left(\sum_{k=1}^{\infty}\left(\frac{n_{k+1}-n_{k}}{n_{k+1}}\right)^{2}\right)^{\frac{1}{2}}\|f\|_{\infty}+\left(\sum_{k=1}^{\infty}\left(\left(\frac{n_{k+1}-n_{k}}{n_{k} n_{k+1}}\right) n_{k}\right)^{2}\right)^{\frac{1}{2}}\|f\|_{\infty} \\
& \leq\left(\sum_{k=1}^{\infty}\left(\frac{n_{k+1}-n_{k}}{n_{k+1}}\right)^{2}\right)^{\frac{1}{2}}\|f\|_{\infty}+\left(\sum_{k=1}^{\infty}\left(\frac{n_{k+1}-n_{k}}{n_{k+1}}\right)^{2}\right)^{\frac{1}{2}}\|f\|_{\infty} \\
& =2\left(\sum_{k=1}^{\infty}\left(\frac{n_{k+1}-n_{k}}{n_{k+1}}\right)^{2}\right)^{\frac{1}{2}}\|f\|_{\infty} .
\end{aligned}
$$

Since $\sum_{k=1}^{\infty}\left(\frac{n_{k+1}-n_{k}}{n_{k+1}}\right)^{2}<\infty$ and $f \in L^{\infty}$, we have $S f \in L^{\infty}$. Moreover, $\|S f\|_{\infty} \leq C\|f\|_{\infty}$. $=\infty$.

Now we consider the negative results for square functions if $\sum_{k=1}^{\infty}\left|\frac{n_{k+1}-n_{k}}{n_{k+1}}\right|^{2}$

Theorem 2.2. Let $\left(n_{k}\right)$ be an increasing sequence in $\mathbb{N}$. If $\sum_{k=1}^{\infty}\left|\frac{n_{k+1}-n_{k}}{n_{k+1}}\right|^{2}$ $=\infty$, then there is an ergodic non-atomic dynamical system $(X, \Sigma, \mu, T)$ and an $f \in L^{\infty}$ such that $\|S f\|_{\infty}=\infty$. 
In the proof of Theorem 2.2, we need some definitions from probability theory and results from [3]. Additional related material is in [1] and [4].

\section{Proof. First step: Construct a particular $\left(a_{k}\right)$.}

Fix a sequence $\left(a_{k}: k \geq 2\right)$. Let $\epsilon_{j}=1$ for $1 \leq j \leq n_{1}$ and let $\epsilon_{j}=a_{k+1}$ when $n_{k}+1 \leq j \leq n_{k+1}$ for $k \geq 1$.

We are going to choose $\left(a_{k}\right)$ inductively to take the values 0 and 1 . So whatever choices we have made for $a_{l}, l \leq k$, we have $\frac{1}{n_{k}} \sum_{j=1}^{n_{k}} \epsilon_{j} \in[0,1]$. Hence, either $\frac{1}{n_{k}} \sum_{j=1}^{n_{k}} \epsilon_{j} \in[0,1 / 2)$ or $\frac{1}{n_{k}} \sum_{j=1}^{n_{k}} \epsilon_{j} \in[1 / 2,1]$. Therefore, we can choose $a_{k+1}$ such that $\left|a_{k+1}-\frac{1}{n_{k}} \sum_{j=1}^{n_{k}} \epsilon_{j}\right| \geq \frac{1}{2}$.

Second step: Construct $\left(x_{n}: n \in \mathbb{Z}\right)$ IID and a suitable non-atomic ergodic dynamical system $(X, \Sigma, \mu, T)$. Let $\Omega=\{0,1\}$ with probability distribution $P$ giving $P(1)=P(0)=\frac{1}{2}$ and define a map $y_{0}$ from $\Omega$ to $\mathbb{R}$ satisfying $y_{0}(0)=0$ and $y_{0}(1)=1$. We construct the product space $X=\prod_{-\infty}^{\infty} \Omega, \Sigma=\prod_{-\infty}^{\infty} 2^{\{0,1\}}$, and $\mu=\prod_{-\infty}^{\infty} P$.

Assume that $w=\left(w_{k}\right) \in X$. We will also write $w(k)$ for the $k^{t h}$ coordinate $w_{k}$. We define a shift operator $T$ on $X$ by $T(w)(n)=w_{n+1}$. We define a random variable $x_{n}(w)=x_{n}\left(\left(w_{k}\right)\right)$ :

$$
x_{n}\left(\left(w_{k}\right)\right)=y_{0}\left(w_{n}\right)= \begin{cases}0 & \text { if } w_{n}=0, \\ 1 & \text { if } w_{n}=1 .\end{cases}
$$

Then, $\left(x_{n}: n \in \mathbb{Z}\right)$ is an IID sequence of $\{0,1\}$-valued random variables on $(X, \Sigma, \mu)$. Also, $(X, \Sigma, \mu, T)$ is a non-atomic ergodic dynamical system.

Third step: Prove $\left\|S x_{0}\right\|_{\infty}=\infty$. Since $T$ is defined as the shift operator on the above non-atomic ergodic dynamical system $(X, \Sigma, \mu, T)$, we have $x_{j} \circ T=x_{j+1}$. Also, $A_{n} x_{0}(w)=\frac{1}{n} \sum_{j=1}^{n} x_{0} \circ T^{j}(w)=\frac{1}{n} \sum_{j=1}^{n} x_{j}(w)$.

Take the $\{0,1\}$-valued sequence $\left(a_{j}\right)$ constructed above. Let

$$
E_{k}=\bigcap_{j=n_{k}+1}^{n_{k+1}}\left\{w \in X: x_{j}(w)=a_{k+1}\right\},
$$

and

$$
F_{M}=\bigcap_{k=1}^{M} E_{k} .
$$

By independence of $\left(x_{j}\right)$, we have $\mu\left(F_{M}\right)>0$ for all $M \geq 1$. Now we calculate the difference of $A_{n_{k}} x_{0}(w)$ and $A_{n_{k+1}} x_{0}(w)$ for $w \in F_{M}$ and $1 \leq k \leq M$. We have

$$
\begin{aligned}
\left|A_{n_{k}} x_{0}(w)-A_{n_{k+1}} x_{0}(w)\right| & =\left|\frac{1}{n_{k+1}} \sum_{j=n_{k}+1}^{n_{k+1}} x_{j}(w)-\left(\frac{1}{n_{k}}-\frac{1}{n_{k+1}}\right) \sum_{j=1}^{n_{k}} x_{j}(w)\right| \\
& =\left|\frac{n_{k+1}-n_{k}}{n_{k+1}} a_{k+1}-\left(\frac{n_{k+1}-n_{k}}{n_{k+1} n_{k}}\right) \sum_{j=1}^{n_{k}} \epsilon_{j}\right| \\
& =\left|\frac{n_{k+1}-n_{k}}{n_{k+1}}\left(a_{k+1}-\frac{1}{n_{k}} \sum_{j=1}^{n_{k}} \epsilon_{j}\right)\right| .
\end{aligned}
$$


We have chosen $a_{k+1}$ to be 0 or 1 such that $\left|\left(a_{k+1}-\frac{1}{n_{k}} \sum_{j=1}^{n_{k}} \epsilon_{j}\right)\right| \geq \frac{1}{2}$. Therefore on the measurable set $F_{M}$ with $\mu\left(F_{M}\right)>0$ for any $k, 1 \leq k \leq M$, we have $\left|A_{n_{k}} x_{0}(w)-A_{n_{k+1}} x_{0}(w)\right| \geq \frac{1}{2} \frac{n_{k+1}-n_{k}}{n_{k+1}}$.

Hence, on $F_{M}$ we have the following estimate:

$$
\begin{aligned}
S_{M} x_{0}(w) & =\left(\sum_{k=1}^{M}\left(A_{n_{k}} x_{0}(w)-A_{n_{k+1}} x_{0}(w)\right)^{2}\right)^{\frac{1}{2}} \\
& \geq\left(\sum_{k=1}^{M}\left(\frac{1}{2}\left|\frac{n_{k+1}-n_{k}}{n_{k+1}}\right|\right)^{2}\right)^{\frac{1}{2}} .
\end{aligned}
$$

Because $\sum_{k=1}^{\infty}\left|\frac{n_{k+1}-n_{k}}{n_{k+1}}\right|^{2}=\infty$, for any $L>0$ we can choose large enough $M$ such that $S_{M} x_{0}(w)>L$ for all $w \in F_{M}$. Therefore, $\left\|S x_{0}\right\|_{\infty} \geq\left\|S_{M} x_{0}\right\| \geq L$. But $L$ is arbitrary, $\left\|S x_{0}\right\|_{\infty}=\infty$. Therefore, $\|S f\|=\infty$ for some $f \in L^{\infty}$ in the non-atomic ergodic dynamical system $(X, \Sigma, \mu, T)$.

Now we apply the Calderón Transfer Principle and the Kakutani-Rokhlin Lemma to the previous result. For convenience, we state the Kakutani-Rokhlin Lemma first.

Lemma 2.3 (Kakutani-Rokhlin Lemma [8], [10], and [11]). Let $(X, \Sigma, \mu, T)$ be an ergodic non-atomic dynamical system, $n$ a positive integer, and $\epsilon>0$. Then, there is a measurable set $E \subset X$ and $\mu(E)>0$ such that $E, T E, \ldots, T^{n} E$ are pairwise disjoint and cover $X$ up to a set of measure less than $\epsilon$.

Theorem 2.4. If $\sum_{k=1}^{\infty}\left|\frac{n_{k+1}-n_{k}}{n_{k+1}}\right|^{2}=\infty$, then for any ergodic non-atomic dynamical system $(X, \Sigma, \mu, T)$, there is no $C$ constant such that $\|S f\|_{\infty} \leq C\|f\|_{\infty}$ for any $f \in L^{\infty}$.

Proof. We use contradiction to prove the theorem. Assume that there is a constant $C$ satisfying $\|S f\|_{\infty} \leq C\|f\|_{\infty}$ for some ergodic non-atomic dynamical system $(X, \Sigma, \mu, \widetilde{T})$. Fix $\varphi \in l^{\infty}(N)$. For any fixed $\epsilon>0$ and $n \geq 1$, by the KakutaniRokhlin Lemma, there is a set $E$ and pairwise disjoint $\left\{\widetilde{T}^{k} E: k=0, \pm 1, \ldots, \pm n\right\}$ such that $\mu\left(X \backslash \bigcup_{k=-n}^{n} \widetilde{T}^{k} E\right)<\epsilon$. We define $f_{n}(x) \in L^{\infty}$ as follows:

$$
f_{n}(x)= \begin{cases}\varphi(k) & \text { if } x \in T^{k} E, \text { and } k=0, \pm 1, \ldots, \pm n, \\ 0 & \text { otherwise. }\end{cases}
$$

We have $f_{n} \in L^{\infty},\left\|f_{n}\right\|_{\infty} \leq\left\|\varphi_{n}\right\|_{\infty}$. Indeed, $\left\|f_{n}\right\|_{\infty}$ increases to $\|\varphi\|_{\infty}$ as $n$ tends to $\infty$. But in any case, for any $M$, we have $\left\|S_{M} f_{n}\right\|_{\infty} \leq C\left\|f_{n}\right\|_{\infty} \leq\|\varphi\|_{\infty}$. In addition, it is also clear from the construction that $\left\|S_{M} f_{n}\right\|_{\infty}$ tends to $\left\|S_{M} \varphi\right\|_{\infty}$ as $n$ tends to $\infty$. Hence, $\left\|S_{M} \varphi\right\|_{\infty} \leq C\|\varphi\|_{\infty}$ for all $\varphi \in l^{\infty}(\mathbb{Z})$. The constant $C$ is independent of $M$. So letting $M$ go to infinity, we have for all $\varphi \in l^{\infty}(\mathbb{Z})$,

$$
\|S \varphi\|_{\infty} \leq C\|\varphi\|_{\infty} .
$$

Now, applying the Calderón Transfer Principle, we have for any dynamical system $(X, \Sigma, \mu, \widetilde{S})$ the inequality $\|S f\|_{\infty} \leq C\|f\|_{\infty}$ for this same constant $C$. This contradicts Theorem 2.2 because we are assuming $\sum_{k=1}^{\infty}\left|\frac{n_{k+1}-n_{k}}{n_{k+1}}\right|^{2}=\infty$.

A close examination of the arguments above show that we have proved this next corollary. It will be useful in the next section. 
Corollary 2.5. Given a non-decreasing sequence $\left(n_{k}\right)$, there exists $\varphi \in l^{\infty}(\mathbb{Z})$ such that

$$
\left\|S_{M} \varphi\right\|_{\infty} \geq \frac{1}{3}\left(\sum_{k=1}^{M}\left|\frac{n_{k+1}-n_{k}}{n_{k+1}}\right|^{2}\right)^{\frac{1}{2}}\|\varphi\|_{\infty} .
$$

So, if

$$
\sum_{k=1}^{\infty}\left|\frac{n_{k+1}-n_{k}}{n_{k+1}}\right|^{2}=\infty
$$

then for any $C>0$, there exist $M$ and $\varphi \in l^{\infty}(\mathbb{Z})$ such that $\left\|S_{M} \varphi\right\|_{\infty} \geq C\|\varphi\|_{\infty}$.

Using a standard Baire Category argument in [2], we have the following theorem.

Theorem 2.6. If $\sum_{k=1}^{\infty}\left|\frac{n_{k+1}-n_{k}}{n_{k+1}}\right|^{2}=\infty$, then for any ergodic non-atomic dynamical system $(X, \Sigma, \mu, T)$ there is an $f \in L^{\infty}$ such that $\|S f\|=\infty$. Furthermore, there is actually a dense $G_{\delta}$ set $\Gamma$ in $L^{\infty}$ such that for all $f \in \Gamma$ we have $\|S f\|=\infty$.

Proof. Let $L_{K}=\left\{f \in L^{\infty}:\|S f\|_{\infty} \leq K\right\}$.

Claim 1: $L_{K}$ is a closed set of $L^{\infty}$.

Since $\left\{f \in L^{\infty}:\left\|S_{M} f\right\|_{\infty} \leq K\right\}$ is a closed set in $L^{\infty}$ for each finite $M$, $L_{K}=\bigcap_{M=1}^{\infty}\left\{f \in L^{\infty}:\left\|S_{M} f\right\|_{\infty} \leq K\right\}$ is a closed set in $L^{\infty}$.

Claim 2: $L_{K}$ has no interior.

If Claim 2 is false, then there is $f_{0} \in L_{K}$ and $\delta>0$ such that $f_{0}+\delta f \in L_{K}$ for any $f$ with $\|f\|_{\infty} \leq 1$. We have the following estimate for $\|S f\|_{\infty}$ if $\|f\|_{\infty} \leq 1$ :

$$
\begin{aligned}
\|S f\|_{\infty} & =\left\|\frac{1}{\delta} S\left(\delta f+f_{0}-f_{0}\right)\right\|_{\infty} \\
& \leq \frac{1}{\delta}\left(\left\|S\left(\delta f+f_{0}\right)\right\|_{\infty}+\left\|S\left(f_{0}\right)\right\|_{\infty}\right) \\
& \leq \frac{1}{\delta}(K+K)=\frac{1}{\delta}(2 K) .
\end{aligned}
$$

Therefore $\|S f\|_{\infty} \leq \frac{1}{\delta}(2 K)\|f\|_{\infty}$ for all $f \in L^{\infty}$. This is a contradiction to Theorem 2.4. Hence $L_{K}$ has empty interior.

Let $\Lambda=\bigcup_{K=1}^{\infty} L_{K}$. Then $\Gamma=L^{\infty} \backslash \Lambda$ is a dense $G_{\delta}$ set and not empty by the Baire Category Theorem in the complete metric space $L^{\infty}$. Moreover, for any $f \in \Gamma$, we have $\|S f\|_{\infty}=\infty$.

3. Characterizing the Strong estimates for Lebesgue Derivatives

In this section, we are going to get the same results for Lebesgue derivatives as we did for ergodic averages.

Theorem 3.1. If $\left(\epsilon_{k}\right)$ is decreasing to 0 and

$$
\sum_{k=1}^{\infty}\left|\frac{\epsilon_{k}-\epsilon_{k+1}}{\epsilon_{k}}\right|^{2}<\infty
$$

then there is a constant

$$
C=2\left\{\sum_{k=1}^{\infty}\left|\frac{\epsilon_{k}-\epsilon_{k+1}}{\epsilon_{k}}\right|^{2}\right\}^{\frac{1}{2}}<\infty
$$

such that $\|S f\|_{\infty} \leq C\|f\|_{\infty}$ for $f \in L^{\infty}(\mathbb{R})$. 
Proof. Let $f \in L^{\infty}(\mathbb{R})$. We have

$$
\begin{aligned}
S f(x) & =\left(\sum_{k=1}^{\infty}\left|\frac{1}{\epsilon_{k+1}} \int_{0}^{\epsilon_{k+1}} f(x+t) d t-\frac{1}{\epsilon_{k}} \int_{0}^{\epsilon_{k}} f(x+t) d t\right|^{2}\right)^{\frac{1}{2}} \\
& =\left(\sum_{k=1}^{\infty}\left|\left(\frac{1}{\epsilon_{k+1}}-\frac{1}{\epsilon_{k}}\right) \int_{0}^{\epsilon_{k+1}} f(x+t) d t-\frac{1}{\epsilon_{k}} \int_{\epsilon_{k+1}}^{\epsilon_{k}} f(x+t) d t\right|^{2}\right)^{\frac{1}{2}} \\
& \leq\left(\sum_{k=1}^{\infty}\left|\left(\frac{1}{\epsilon_{k+1}}-\frac{1}{\epsilon_{k}}\right) \int_{0}^{\epsilon_{k+1}} f(x+t) d t\right|^{2}+\left.\left|\sum_{k=1}^{\frac{1}{2}}\right| \frac{1}{\epsilon_{k}} \int_{\epsilon_{k+1}}^{\epsilon_{k}} f(x+t) d t\right|^{2}\right)^{\frac{1}{2}} \\
& \leq\left(\sum_{k=1}^{\infty}\left|\frac{\epsilon_{k}-\epsilon_{k+1}}{\epsilon_{k} \epsilon_{k+1}} \epsilon_{k+1}\right|^{2}\right)^{\frac{1}{2}}\|f\|_{\infty}+\left(\sum_{k=1}^{\infty}\left|\frac{\epsilon_{k}-\epsilon_{k+1}}{\epsilon_{k}}\right|^{2}\right)^{\frac{1}{2}}\|f\|_{\infty} \\
& \leq\left(\sum_{k=1}^{\infty}\left|\frac{\epsilon_{k}-\epsilon_{k+1}}{\epsilon_{k}}\right|^{2}\right)^{\frac{1}{2}}\|f\|_{\infty}+\left(\sum_{k=1}^{\infty} \mid\left(\left.\left.\frac{\epsilon_{k}-\epsilon_{k+1}}{\epsilon_{k}}\right|^{2}\right|^{\frac{1}{2}}\|f\|_{\infty}\right.\right. \\
& =2\left(\sum_{k=1}^{\infty}\left|\frac{\epsilon_{k}-\epsilon_{k+}}{\epsilon_{k}}\right|^{2}\right)^{\frac{1}{2}}\|f\|_{\infty} .
\end{aligned}
$$

Because $\sum_{k=1}^{\infty}\left(\frac{\epsilon_{k}-\epsilon_{k+1}}{\epsilon_{k}}\right)^{2}<\infty$, we have $S f \in L^{\infty}$.

We can also prove the converse result for this bound, analogously to the arguments in the last section. First, we need a suitable variant of Corollary 2.5.

Lemma 3.2. Given a non-decreasing sequence $\left(n_{k}\right)$, there exists $\varphi \in L^{\infty}(\mathbb{R})$ such that $\left\|S_{M} \varphi\right\|_{\infty} \geq \frac{1}{3}\left(\sum_{k=1}^{M}\left|\frac{n_{k+1}-n_{k}}{n_{k+1}}\right|^{2}\right)^{\frac{1}{2}}\|\varphi\|_{\infty}$. So, if $\sum_{k=1}^{\infty}\left|\frac{n_{k+1}-n_{k}}{n_{k+1}}\right|^{2}=\infty$, then for any $C>0$, there exist $M$ and $\varphi \in L^{\infty}(\mathbb{R})$ such that $\left\|S_{M} \varphi\right\|_{\infty} \geq C\|\varphi\|_{\infty}$.

Proof. The proof of this is a standard quantization argument. A more complete version of this result allows one to transfer back and forth between norm inequalities in $l^{\infty}(\mathbb{Z})$ for ergodic averages for the shift $T(i)=i+1, i \in \mathbb{Z}$, to norm inequalities in $L^{\infty}(\mathbb{R})$ for the ergodic averages given by the regular flow of $\mathbb{R}$ on itself. We only need one direction of this here. We will be comparing values of two square functions, one from averages on $\mathbb{Z}$ and one from averages on $\mathbb{R}$. So we will denote the first square function by $\mathcal{S}$ and the second one just by $S$ as we have been doing in this section. Let $\varphi_{o} \in l^{\infty}(\mathbb{Z})$ be as in Corollary 2.5 such that $\left\|\mathcal{S}_{M} \varphi_{o}\right\|_{\infty} \geq$ $\frac{1}{3}\left(\sum_{k=1}^{M}\left|\frac{n_{k+1}-n_{k}}{n_{k+1}}\right|^{2}\right)^{\frac{1}{2}}\left\|\varphi_{o}\right\|_{\infty}$. Let $\varphi \in L^{\infty}(\mathbb{R})$ be given by $\varphi=\varphi_{o}^{*} \star 1_{[0,1]}$. So $\varphi$ takes the value $\overline{\varphi_{o}}(-k)$ on $[k, k+1)$. Also, $\|\varphi\|_{\infty}=\left\|\varphi_{o}\right\|_{\infty}$. Consider a typical average in $\mathbb{R}$ given by $A_{n} \varphi(t)=\frac{1}{n} \int_{0}^{n} \varphi(t+x) d x$. This is the same as $\mathcal{A}_{n} \varphi_{o}^{*} \star 1_{[-1,0]} \star 1_{[0,1]}$ where $\mathcal{A}_{n} \varphi_{o}(j)=\frac{1}{n} \sum_{k=0}^{n-1} \varphi_{o}(j+k)$ for all $j \in \mathbb{Z}$. Now $T(t)=1_{[-1,0]} \star 1_{[0,1]}$ is a continuous piecewise linear function that is 0 off $(-1,1), 1$ at 0 , and linear on each of $[-1,0]$ and $[0,1]$. This creates some interference between adjacent values when trying to make the estimate that we need. For example, for $t$ near $m \in \mathbb{Z}$, $A_{n} \varphi(t)=\delta_{t} \mathcal{A}_{n} \varphi_{o}^{*}(m)+\epsilon_{t, 1} \mathcal{A}_{n} \varphi_{o}^{*}(m-1)+\epsilon_{t, 2} \mathcal{A}_{n} \varphi_{o}^{*}(m+1)$ where $\delta_{t}$ approaches 1 and both $\epsilon_{t, 1}$ and $\epsilon_{t, 2}$ approach 0 as $t$ converges to $m$. Hence, for values of $t$ 
close enough to $m, S_{M}^{\left(n_{k}\right)} \varphi(t)$ is as close to $\mathcal{S}_{M}^{\left(n_{k}\right)} \varphi_{o}(-m)$ as we like. Therefore, $\left\|S_{M}^{\left(n_{k}\right)} \varphi\right\|_{\infty} \geq\left\|\mathcal{S}_{M}^{\left(n_{k}\right)} \varphi_{o}\right\|_{\infty} \geq \frac{1}{3}\left(\sum_{k=1}^{M}\left|\frac{n_{k+1}-n_{k}}{n_{k+1}}\right|^{2}\right)^{\frac{1}{2}}\left\|\varphi_{o}\right\|_{\infty}$. This gives the desired result.

Theorem 3.3. If $\left\{\epsilon_{k}\right\}_{k=1}^{\infty}$ is decreasing to 0 and $\sum_{k=1}^{\infty}\left|\frac{\epsilon_{k}-\epsilon_{k+1}}{\epsilon_{k}}\right|^{2}=\infty$, there is an $f \in L^{\infty}$ such that $\|S f\|_{\infty}=\infty$. Indeed, there is a dense $G_{\delta}$ set $\Gamma$ in $L^{\infty}$ such that $\|S f\|_{\infty}=\infty$ for any $f \in \Gamma$.

Proof. Let $C_{0}=4 \sum_{n=1}^{\infty} \frac{1}{n^{2}}$.

First step: For any $C>0$, there is an $M \in \mathbb{Z}$ and a $\varphi \in L^{\infty}$ such that $\left\|S_{M}^{\left\{r_{k}\right\}} \varphi\right\|_{\infty} \geq C\|\varphi\|_{\infty}$. Fix a large value $\widetilde{C}$ that we will specify later. Choose numbers $H, M$, and $\left\{r_{k}\right\}_{k=1}^{M}$ such that $r_{k}=\frac{H}{\epsilon_{M}} \epsilon_{M-k+1}$, there is only one $r_{k}$ in the interval $\left[n_{k}, n_{k}+1\right]$ where $n_{k}=\left[r_{k}\right]$, and $\sum_{k=1}^{M}\left|\frac{\epsilon_{k}-\epsilon_{k+1}}{\epsilon_{k}}\right|^{2} \geq \widetilde{C}$. We have $r_{k}=n_{k}+\alpha_{k}$ where $0 \leq \alpha_{k}<1$. Since

$$
\begin{aligned}
\sum_{k=1}^{M}\left|\frac{r_{k+1}-r_{k}}{r_{k+1}}\right|^{2} & =\sum_{k=1}^{M}\left|\frac{\frac{H}{\epsilon_{M}} \epsilon_{M-k}-\frac{H}{\epsilon_{M}} \epsilon_{M-k+1}}{\frac{H}{\epsilon_{M}} \epsilon_{M-k}}\right|^{2} \\
& =\sum_{k=1}^{M}\left|\frac{\epsilon_{M-k}-\epsilon_{M-k+1}}{\epsilon_{M-k}}\right|^{2}=\sum_{k=1}^{M}\left|\frac{\epsilon_{k}-\epsilon_{k+1}}{\epsilon_{k}}\right|^{2},
\end{aligned}
$$

we have $\sum_{k=1}^{M}\left|\frac{r_{k+1}-r_{k}}{r_{k+1}}\right|^{2} \geq \widetilde{C}$ as well.

We calculate the difference of the terms $\frac{n_{k+1}-n_{k}}{n_{k+1}}$ and $\frac{r_{k+1}-r_{k}}{r_{k+1}}$ :

$$
\begin{aligned}
\left|\frac{n_{k+1}-n_{k}}{n_{k+1}}-\frac{r_{k+1}-r_{k}}{r_{k+1}}\right| & =\left|\frac{n_{k} r_{k+1}-n_{k+1} r_{k+1}-n_{k+1} r_{k}+n_{k+1} r_{k+1}}{n_{k+1} r_{k+1}}\right| \\
& =\left|\frac{n_{k} r_{k+1}-n_{k+1} r_{k}}{n_{k+1} r_{k+1}}\right| \\
& =\left|\frac{n_{k}\left(n_{k+1}+\alpha_{k+1}\right)-n_{k+1}\left(n_{k}+\alpha_{k}\right)}{n_{k+1} r_{k+1}}\right| \\
& =\left|\frac{n_{k} \alpha_{k+1}-n_{k+1} \alpha_{k}}{n_{k+1} r_{k+1}}\right| \\
& \leq\left|\frac{2 n_{k+1}}{n_{k} n_{k+1}}\right| \\
& =\left|\frac{2}{n_{k}}\right| \leq\left|\frac{2}{k}\right| .
\end{aligned}
$$

Now we estimate the difference between $\left|\frac{n_{k+1}-n_{k}}{n_{k+1}}\right|^{2}$ and $\left|\frac{r_{k+1}-r_{k}}{r_{k+1}}\right|^{2}$ :

$$
\begin{aligned}
\left|\left(\frac{n_{k+1}-n_{k}}{n_{k+1}}\right)^{2}-\left(\frac{r_{k+1}-r_{k}}{r_{k+1}}\right)^{2}\right| & \leq\left|\frac{n_{k+1}-n_{k}}{n_{k+1}}-\frac{r_{k+1}-r_{k}}{r_{k+1}}\right|^{2} \\
& \leq\left|\frac{2}{k}\right|^{2}=\frac{4}{k^{2}}
\end{aligned}
$$


Therefore, we have $\left(\frac{n_{k+1}-n_{k}}{n_{k+1}}\right)^{2} \geq\left(\frac{r_{k+1}-r_{k}}{r_{k+1}}\right)^{2}-\frac{4}{k^{2}}$. Hence,

$$
\sum_{k=1}^{M}\left(\frac{n_{k+1}-n_{k}}{n_{k+1}}\right)^{2} \geq \sum_{k=1}^{M}\left(\frac{r_{k+1}-r_{k}}{r_{k+1}}\right)^{2}-\sum_{k=1}^{M} \frac{4}{k^{2}} \geq \widetilde{C}-C_{0} .
$$

According to Lemma 3.2, we have a $\varphi \in L^{\infty}(\mathbb{R})$ such that the partial sum $\left\|S_{M}^{\left(n_{k}\right)} \varphi\right\|_{\infty}$ $\geq \frac{1}{3}\left(\widetilde{C}-C_{0}\right)^{\frac{1}{2}}\|\varphi\|_{\infty}$.

Now we will transfer the partial sum results for the sequence $\left\{n_{k}\right\}_{k=1}^{M}$ to the sequence $\left\{r_{k}\right\}_{k=1}^{M}$ with some adjustment for the constants. We are going to prove that for the above $\varphi \in L^{\infty}(\mathbb{R})$ we have $\left\|S_{M}^{\left(r_{k}\right)} \varphi\right\|_{\infty} \geq \frac{1}{3}\left(\left(\widetilde{C}-C_{0}\right)^{\frac{1}{2}}-\sqrt{C_{0}}\right)\|\varphi\|_{\infty}$.

First we estimate the difference between $\frac{1}{r_{k}} \int_{0}^{r_{k}} \varphi(x+t) d t$ and $\frac{1}{n_{k}} \int_{0}^{n_{k}} \varphi(x+t) d t$. Notice that $0 \leq r_{k}-n_{k}<1$. We have

$$
\begin{aligned}
\mid \frac{1}{r_{k}} \int_{0}^{r_{k}} \varphi(x+t) d t-\frac{1}{n_{k}} \int_{0}^{n_{k}} & \varphi(x+t) d t \mid \\
& =\left|\left(\frac{1}{r_{k}}-\frac{1}{n_{k}}\right) \int_{0}^{n_{k}} \varphi(x+t) d t-\frac{1}{r_{k}} \int_{n_{k}}^{r_{k}} \varphi(x+t) d t\right| \\
& =\left|\frac{r_{k}-n_{k}}{r_{k} n_{k}}\right|\|\varphi\|_{\infty}+\left|\frac{r_{k}-n_{k}}{r_{k}}\right|\|\varphi\|_{\infty} \\
& \leq \frac{1}{r_{k}}\|\varphi\|_{\infty}+\frac{1}{r_{k}}\|\varphi\|_{\infty} \leq \frac{2}{k}\|\varphi\|_{\infty} .
\end{aligned}
$$

Now we are ready to estimate the difference between $S_{M}^{\left(r_{k}\right)} \varphi(x)$ and $S_{M}^{\left\{n_{k}\right\}} \varphi(x)$.

$$
\begin{aligned}
\left|S_{M}^{\left\{r_{k}\right\}} \varphi(x)-S_{M}^{\left\{n_{k}\right\}} \varphi(x)\right|=\mid\left\{\sum_{k=1}^{M}\left(\frac{1}{r_{k}} \int_{0}^{r_{k}} \varphi(x+t) d t-\frac{1}{r_{k+1}} \int_{0}^{r_{k+1}} \varphi(x+t) d t\right)^{2}\right\}^{\frac{1}{2}} \\
\quad-\left\{\sum_{k=1}^{M}\left(\frac{1}{n_{k}} \int_{0}^{n_{k}} \varphi(x+t) d t-\frac{1}{n_{k+1}} \int_{0}^{\frac{1}{2}} \varphi(x+t) d t\right)^{n_{k+1}} \mid\right. \\
\leq \\
\quad\left\{\sum _ { k = 1 } ^ { M } \left(\left(\frac{1}{r_{k}} \int_{0}^{r_{k}} \varphi(x+t) d t-\frac{1}{n_{k}} \int_{0}^{n_{k}} \varphi(x+t) d t\right)\right.\right. \\
\left.\left.\quad-\left(\frac{1}{r_{k+1}} \int_{0}^{r_{k+1}} \varphi(x+t) d t-\frac{1}{n_{k+1}} \int_{0}^{n_{k+1}} \varphi(x+t) d t\right)\right)^{2}\right\}^{\frac{1}{2}} \\
\leq 2\left\{\sum_{k=1}^{M}\left(\frac{2}{k}\|\varphi\|_{\infty}\right)^{2}\right\}^{\frac{1}{2}}=4\left(\sum_{k=1}^{M} \frac{1}{k^{2}}\right)^{\frac{1}{2}}\|\varphi\|_{\infty} .
\end{aligned}
$$


Taking the supremum over all $x \in \mathbb{R}$, we have $\left\|S_{M}^{\left\{r_{k}\right\}} \varphi-S_{M}^{\left\{n_{k}\right\}} \varphi\right\|_{\infty} \leq 2 \sqrt{C_{0}}\|\varphi\|_{\infty}$. So we have

$$
\left|\left\|S_{M}^{\left(r_{k}\right)} \varphi\right\|_{\infty}-\left\|S_{M}^{\left(n_{k}\right)} \varphi\right\|_{\infty}\right| \leq 2 \sqrt{C_{0}}\|\varphi\|_{\infty} .
$$

Hence, we have $\left\|S_{M}^{\left(r_{k}\right)} \varphi\right\|_{\infty} \geq\left\|S_{M}^{\left(n_{k}\right)} \varphi\right\|_{\infty}-2 \sqrt{C_{0}}\|\varphi\|_{\infty}$. Finally, we have

$$
\left\|S_{M}^{\left(r_{k}\right)} \varphi\right\|_{\infty} \geq \frac{1}{3}\left(\left(\widetilde{C}-C_{0}\right)^{\frac{1}{2}}-2 \sqrt{C_{0}}\right)\|\varphi\|_{\infty}
$$

But then since $C_{0}$ is an absolute constant and $\widetilde{C}$ is arbitrary, it is clear that for all $C$ there exist $M$ and $\varphi \in L^{\infty}(\mathbb{R})$ such that $\left\|S_{M}^{\left(r_{k}\right)} \varphi\right\|_{\infty} \geq C\|\varphi\|_{\infty}$

Second step: For any $C>0$, there is a $g \in L^{\infty}$ such that $\left\|S^{\left(\epsilon_{k}\right)} g\right\|_{\infty} \geq$ $C\|g\|_{\infty}$. In the first step, we have $M$ and $\varphi \in L^{\infty}(\mathbb{R})$ such that $\left\|S_{M}^{\left(r_{k}\right)} \varphi\right\|_{\infty} \geq$ $C\|\varphi\|_{\infty}$. We let $g(x)=\varphi\left(\frac{H}{\epsilon_{M}} x\right)$ and note that $\|g\|_{\infty}=\|\varphi\|_{\infty}$. We calculate

$$
\begin{aligned}
\frac{1}{\epsilon_{k}} \int_{0}^{\epsilon_{k}} g(x+t) d t & =\frac{1}{\epsilon_{k}} \int_{0}^{\epsilon_{k}} \varphi\left(\frac{H}{\epsilon_{M}}(x+t)\right) d t \\
& =\frac{1}{\epsilon_{k}} \int_{0}^{\epsilon_{k}} \varphi\left(\frac{H}{\epsilon_{M}} x+\frac{H}{\epsilon_{M}} t\right) d t \\
& =\frac{1}{\epsilon_{k}} \int_{0}^{\frac{H}{\epsilon_{M}} \epsilon_{k}} \varphi\left(\frac{H}{\epsilon_{M}} x+u\right) \frac{1}{\frac{H}{\epsilon_{M}}} d u \\
& =\frac{1}{\frac{H}{\epsilon_{M}} \epsilon_{k}} \int_{0}^{\frac{H}{\epsilon_{M}} \epsilon_{k}} \varphi\left(\frac{H}{\epsilon_{M}} x+u\right) d u \\
& =\frac{1}{r_{M-k+1}} \int_{0}^{r_{M-k+1}} \varphi\left(\frac{H}{\epsilon_{M}} x+u\right) d u .
\end{aligned}
$$

So we have $S_{M}^{\left\{\epsilon_{k}\right\}} g(x)=S_{M}^{\left\{r_{k}\right\}} \varphi\left(\frac{H}{\epsilon_{M}} x\right)$. Thus, $\left\|S_{M}^{\left\{\epsilon_{k}\right\}} g\right\|_{\infty}=\left\|S_{M}^{\left\{r_{k}\right\}} \varphi\right\|_{\infty}$. Hence, we have $\left\|S_{M}^{\left\{\epsilon_{k}\right\}} g\right\|_{\infty} \geq C\|g\|_{\infty}$. Because $\left\|S^{\left(\epsilon_{k}\right)} g\right\|_{\infty} \geq\left\|S_{M}^{\left\{\epsilon_{k}\right\}} g\right\|_{\infty}$, we have our results for the second step $\left\|S^{\left(\epsilon_{k}\right)} g\right\|_{\infty} \geq C\|g\|_{\infty}$.

Third step: There exists a generic set of $f \in L^{\infty}$ such that $\left\|S^{\left(\epsilon_{k}\right)} f\right\|_{\infty}=\infty$. This argument is a Baire Category argument that is analogous to the one given in Theorem 2.6.

The same results as in Theorem 3.3 hold for $R_{k}$ increasing to infinity in place of $\epsilon_{k}$ decreasing to zero. In this context, also see [12] where important caveats about square functions are pointed out; see the example on p. 245 for instance. In [9], for $\epsilon_{n}=2^{-n}$, there is a detailed discussion for square functions in ergodic averages and Lebesgue derivatives. 


\section{Further RESUlts}

The methods above can be used on other operators. Let $B=\left(B_{n}\right)$ be a sequence of linear operators on $L^{p}$ and let $\left(B_{n_{k}}\right)$ be a fixed subsequence. For any $r, 1 \leq r<$ $\infty$, let

$$
S_{r}^{\left(n_{k}\right)} f=\left(\sum_{k=1}^{\infty}\left|B_{n_{k+1}} f-B_{n_{k}} f\right|^{r}\right)^{1 / r}
$$

and let

$$
O_{r}^{\left(n_{k}\right)} f=\left(\sum_{k=1}^{\infty} \sup _{n_{k} \leq i, j \leq n_{k+1}}\left|B_{j} f-B_{i} f\right|^{r}\right)^{1 / r} .
$$

The following result holds; the proof is similar to arguments in the previous sections.

Theorem 4.1. If $\left(B_{n}\right)=\left(A_{n}\right)$, the standard ergodic averages for an ergodic dynamical system, or if $\left(B_{n}\right)=\left(D_{n}\right)$ for some sequence $\left(\epsilon_{n}\right)$ decreasing to 0 , then $S_{r}^{\left(n_{k}\right)}$ and $O_{r}^{\left(n_{k}\right)}$ both map $L^{\infty}$ to $L^{\infty}$ if and only if $\sum_{k=1}^{\infty}\left(\frac{n_{k+1}-n_{k}}{n_{k}}\right)^{r}<\infty$. If $\sum_{k=1}^{\infty}\left(\frac{n_{k+1}-n_{k}}{n_{k}}\right)^{r}=\infty$, then there is a dense $G_{\delta}$ subset $\Gamma$ of $L^{\infty}$ such that for all $f \in \Gamma$, both $\left\|S_{r}^{\left(n_{k}\right)} f\right\|_{\infty}=\infty$ and $\left\|O_{r}^{\left(n_{k}\right)} f\right\|_{\infty}=\infty$.

Remark 4.2. This remark is about general results for operators $S_{r}, O_{r}$, and $V_{r}$ with $1 \leq r<\infty$.

(1) It is not hard to see that a given $\left(n_{k}\right)$ might not allow $S_{r}^{\left(n_{k}\right)}$ or $O_{r}^{\left(n_{k}\right)}$ to map $L^{\infty}$ to $L^{\infty}$, but for larger values of $r$ they would map $L^{\infty}$ to $L^{\infty}$.

(2) Consider

$$
V_{r}^{B} f=\left(\sup _{\left(n_{k}\right)} \sum_{k=1}^{\infty}\left|B_{n_{k+1}} f-B_{n_{k}} f\right|^{r}\right)^{1 / r}
$$

where the supremum is over all non-decreasing subsequences. Because of the supremum over all non-decreasing sequences in the definition, $V_{r}^{B}$ never maps $L^{\infty}$ to $L^{\infty}$

\section{REFERENCES}

1. Y. S. Chow and H. Teicher, Probability theory: independence, interchangeability, martingales, Springer-Verlag, New York, U.S.A., 1988. MR953964 (89e:60001)

2. J. B. Conway, A course in functional analysis, Springer, New York, U.S.A., 1990. MR1070713 (91e:46001)

3. R. Durrett, Probability theory and examples, Duxbury Press, New York, U.S.A., 1996. MR1609153 (98m:60001)

4. A. Garsia, Martingale inequalities: seminar notes on recent progress, W. A. Benjamin Inc., Reading, MA, 1973. MR0448538 (56:6844)

5. R. Jones, I. Ostrovskii and J. Rosenblatt. Square functions in ergodic theory, Ergodic Theory and Dynamical Systems, 16, 267-305, 1996. MR1389625 (97f:28044)

6. R. Jones, R. Kaufman, J. M. Rosenblatt and M. Wierdl, Oscillation in ergodic theory, Ergodic Theory and Dynamical Systems, 18, 889-935, 1998. MR1645330 (2000b:28019)

7. R. Jones, J. Rosenblatt and Mate Wierdl, Oscillation in ergodic theory: higher dimensional results, Israel Journal of Math., 135, 1-27, 2003. MR1996394 (2004h:37005)

8. S. Kakutani, Induced measure-preserving transformations, Pro. Japan Acad., 19, 41-635, 1943. MR0014222 (7:255f)

9. C. Liu, The convergence of Lebesgue derivatives and ergodic averages, Thesis, University of Illinois at Urbana-Champaign, 2005.

10. K. Petersen, Ergodic Theory, Cambridge University Press. 1983. MR833286 (87i:28002) 
11. V. A. Rokhlin, A 'general' measure-preserving transformation is not mixing, Dokl. Akad. Nauk. SSSR, 60, 51-349, 1948.

12. A. De La Torre and J. L. Torrea, One-sided discrete square function, Studia Mathematica, 153, 243-260, 2003. MR1978442 (2004a:42015)

Department of Mathematics and Statistics, Wallace 313, Eastern Kentucky UniverSity, Richmond, Kentucky 40475

E-mail address: mary.liu@eku.edu

Department of Mathematics, University of Illinois at Champaign-Urbana, 1409 W. Green Street, Urbana, Illinois 61801-2975

E-mail address: jrsnbltt@math.uiuc.edu 